\title{
Sintomas do trato digestivo superior e distúrbios motores do esôfago em pacientes portadores da forma indeterminada da doença de Chagas crônica
}

\author{
Upper gastrointestinal symptoms and esophageal motility disorders \\ in indeterminate Chagas' disease patients
}

\author{
Rafaela de Liz P. Sanchez-Lermen¹, Eduardo Dick², José Augusto Pádua Salas² \\ e Cor Jesus Fernandes Fontes ${ }^{1,2}$
}

\begin{abstract}
RESUMO
o estudo descreve os sintomas referidos por portadores da forma indeterminada da doença de Chagas crônica e avalia sua associação com alterações da motilidade esofágica. Manometria do esôfago foi realizada em 50 pacientes, medindo-se a extensão e a pressão do esfíncter inferior do esôfago, o peristaltismo e a amplitude de contração do corpo esofágico. Oito (16\%) pacientes apresentaram relaxamento parcial do esfincter inferior, 13 (26\%) apresentaram aperistalse parcial e 20 (40\%) apresentaram hipocontratilidade no esôfago distal. Sintomas digestivos altos foram referidos por 24 (48\%) pacientes, sendo mais freqüentes a pirose, a regurgitação e o desconforto intermitente à deglutição. Esses sintomas foram referidos por 17 (51,5\%) de 33 pacientes com alterações motoras do esôfago e por 7 (41,2\%) de 17 pacientes com manometria normal, diferença essa não estatisticamente significante $(p=0,69)$. Esses achados sugerem que portadores da FCI apresentam sintomas inespecíficos do trato digestivo superior que podem dificultar a sua classificação com base apenas no exame clínico e radiológico, e que é alta a frequiência de portadores desta forma que apresentam distúrbios motores do esôfago.
\end{abstract}

Palavras-chaves: Doença de Chagas. Esofagopatia chagásica. Esôfago. Manometria.

\section{ABSTRACT}

This study describes the symptoms reported by patients with the indeterminate form of chronic Chagas' disease and evaluates associations between these symptoms and alterations in esophageal motility. Esophageal manometry was performed on 50 patients, with measurements of the length and pressure of the lower esophageal sphincter, peristaltism and the amplitude of contractions of the esophageal body. Eight (16\%) patients presented partial relaxation of the lower sphincter, 13 (26\%) presented partial lack of peristalsis and 20 (40\%) presented hypocontractility of the distal esophagus. Upper digestive symptoms were reported by 24 (48\%) patients, and the most frequent of these were heartburn, regurgitation and intermittent discomfort when swallowing. Such symptoms were reported by 17 (51.5\%) of the 33 patients with esophageal motor disorders and by 7 (41.2\%) of the 17 patients with normal manometry, which was not a statistically significant difference $(p=0.69)$. These findings suggest that patients with the indeterminate form of Chagas' disease present nonspecific symptoms in the upper digestive tract that may make it difficult to classify the disease solely on the basis of radiological and clinical examination. Furthermore, a high proportion of patients with this form present esophageal motor disorders.

Key-words: Chagas' disease. Chagasic esophagopathy. Esophagus. Manometry.

A doença de Chagas no trato digestivo é caracterizada por lesão dos plexos nervosos intramurais em virtude do parasitismo das camadas musculares vizinhas, repercutindo na função motora, principalmente do esôfago e do cólon, fazendo com que a musculatura lisa desses órgãos responda com contrações desordenadas e hiperreativas, ou hiporreativas, na dependência do estímulo que é feito ao órgão. A desnervação resultante da lesão neural tem intensidade variável, irregular, imprevisível e acomete diferentes segmentos desses órgãos ${ }^{1730}$.

Após a fase aguda da doença, os pacientes passam por longo período assintomático (10 a 30 anos), sem evidência de complicação, denominados de forma indeterminada da doença

1. Curso de Pós-graduação em Ciências da Saúde, Faculdade de Ciências Médicas, Universidade Federal de Mato Grosso, Cuiabá, Mato Grosso. 2. Núcleo de Estudos de Doenças Infecciosas e Tropicais de Mato Grosso, Faculdade de Ciências Médicas, Universidade Federal de Mato Grosso, Cuiabá, MT.

Endereço para correspondência: Dra. Rafaela de Liz Pellegrim Sanchez Lermen. Rua Comandante Costa 1701/84, $78020-400$ Cuiabá MT, Brasil.

Tel: 5565 3028-5252; Fax: 5565 3624-2292

e-mail: rlslermen@terra.com.br

Recebido para publicação em 12/6/2006

Aceito em 8/2/2007 
de Chagas crônica (FCI) ou forma latente. Esta forma caracterizase pela positividade de exames sorológicos e/ou parasitológicos para a doença de Chagas, ausência de sintomas e/ou sinais da doença e resultados normais aos exames eletrocardiográfico, radiológico do coração, esôfago e cólon, conforme estabelecido pela I Reunião de Pesquisa Aplicada em doença de Chagas de Araxá em $1984^{26}$.

A complicação característica do comprometimento esofágico pela doença de Chagas crônica, o megaesôfago, assim como o aparecimento de sintomas típicos como disfagia, regurgitação e emagrecimento podem ocorrer após vários anos do diagnóstico sorológico e/ou do estabelecimento da lesão neuromotora. Assim, entre a situação de esôfago normal e de megaesôfago, com inervação quase totalmente destruída, um número variado de situações intermediárias existe ${ }^{13} \mathrm{e}$, portanto, outros sintomas relacionados à lesão esofágica podem se manifestar, muitos deles presentes em diversas doenças, o que dificulta a interpretação etiológica como decorrente de complicação chagásica.

A grande prevalência de pacientes portadores da FCI no Brasil ${ }^{1}$, muitas vezes apresentando queixas inespecíficas relativas ao trato digestivo superior, leva à angústia diagnóstica na identificação de comprometimento esofágico apenas pelos exames clínico e radiológico. Se tais sintomas são resultantes de algum comprometimento esofágico provocado pela doença de Chagas, é fundamental que essa etiologia seja identificada, para a garantia de um melhor acompanhamento do paciente. 0 emprego de técnicas de diagnóstico funcional do esôfago que possam identificar e quantificar distúrbios motores de diferentes intensidades em portadores da FCI poderá permitir uma possível avaliação etiológica dos sintomas do trato digestivo superior por eles referido, além de contribuir para a caracterização de alterações motoras sugestivas e precoces do comprometimento esofágico da doença.

O diagnóstico da esofagopatia chagásica é realizado principalmente pelo exame radiológico contrastado do esôfago. Porém, outros exames como a cintilografia de trânsito esofágico e a manometria do esôfago podem ser utilizados para avaliar a sua função motora e identificar complicações iniciais da doença. Ambos são adequados para esse objetivo, uma vez que demonstraram alta concordância de resultados em estudo realizado com pacientes chagásicos ${ }^{28}$.

Neste estudo são descritas as queixas digestivas e as alterações manométricas esofágicas de pacientes portadores da FCI, no intuito de avaliar possível associação entre a presença de sintomas do trato digestivo superior e presença de alterações precoces da motilidade esofágica na doença de Chagas.

\section{PACIENTES E MÉTODOS}

Pacientes. 0 estudo foi realizado no Ambulatório de Infectologia do Hospital Universitário Júlio Müller, da Universidade Federal de Mato Grosso, no período de 2003 a 2005. Foram incluídos 50 pacientes portadores da FCI, sendo 32 (64\%) do sexo feminino e com idade variando de 17 a 59 anos (média \pm DP de
$41,8 \pm 10,6$ anos). Exame clínico sumário foi realizado em todos eles, com ênfase na identificação de sintomas do trato digestivo alto, quais seja, pirose, desconforto à deglutição, disfagia, regurgitação, sialorréia, odinofagia, dor torácica retroesternal. Outras queixas relacionadas ao trato digestivo, como tosse, dispnéia e constipação intestinal crônica, também foram inquiridas. Todos esses sintomas foram devidamente caracterizados quanto à cronicidade, ritmicidade e periodicidade.

O diagnóstico de FCI foi confirmado por exame sorológico positivo em pelo menos duas técnicas convencionais e resultados normais nos estudos eletrocardiográfico e radiológico do coração, esôfago e cólon, além de ausência de sintoma clássico de esofagopatia chagásica, como a disfagia. Exame radiológico normal do esôfago foi definido quando a esofagografia, realizada em posição ortostática, revelou diâmetro esofágico na visão póstero anterior menor que $3 \mathrm{~cm}$, trânsito esofágico ocorrendo em duração inferior a 10 segundos e ausência de retenção do meio de contraste 9 . Endoscopia digestiva alta foi realizada em todos os pacientes que apresentaram sintomas do trato digestivo superior. A indicação do paciente ao estudo manométrico do esôfago obedeceu aos seguintes critérios: 1) ser portador da FCI; 2) não apresentar evidências clínicas de insuficiência cardíaca, hepática ou renal, doenças sistêmicas do tecido conjuntivo, diabetes mellitus ou alcoolismo; 3) não apresentar, ao exame radiológico do esôfago, evidências de hérnia de hiato diafragmático ou de doenças orgânicas esofágicas, gástrica e/ou duodenais; 4) concordar em participar do estudo e assinar termo de consentimento livre e esclarecido.

Estudo manométrico do esôfago. Foi utilizada uma sonda de polivinil de $4,5 \mathrm{~mm}$ de diâmetro externo, contendo em seu interior oito canais de $0,8 \mathrm{~mm}$ de diâmetro interno (Zinetics Medical, Inc. Zinetics Manometric Catheters, Stockholm, Sweden). As aberturas laterais dos quatro canais proximais da sonda distanciam-se entre si em $5 \mathrm{~cm}$, formando ângulos de $90^{\circ}$ entre elas e foram utilizadas para a avaliação da pressão e coordenação do corpo do esôfago. Os quatro canais distais estão localizados em um mesmo nível, também formando ângulos de $90^{\circ}$ entre si e foram utilizados para medir a pressão do esfíncter inferior do esôfago (EIE). Cada canal foi conectado a transdutores de pressão (Modelo pvd Medizin technik GmbH, Kirchseeon, Alemanha) com saídas para um polígrafo de oito canais (PC Polygraph HR Synectics Medical, Stockholm, Sweden) e analisado pelo software Esophageal Manometry, version 2.03, 1997 (Synectics Medical, London, UK). Os canais foram perfundidos continuamente com água destilada, a um fluxo de $0,6 \mathrm{ml} / \mathrm{min}$, por meio de um sistema de pressão de baixa complacência, devidamente calibrado de acordo com as recomendações do fabricante do equipamento (Medtronic Synectics BI, MKII Manometric Perfusion Pump Dentsleeve, Sidney, Austrália).

Para o exame manométrico os pacientes, em jejum de 12 horas, foram colocados em decúbito dorsal. A sonda foi introduzida por uma das narinas até o estômago, sob anestesia local com lidocaína geléia a $2 \%$. Pelos quatro canais distais, mediu-se a pressão média intragástrica, considerando-a como pressão zero na linha de base. A pressão de repouso do EIE foi medida em duplicata, pelo método 
de station pull-through (retirada estacionária em intervalos de $1 \mathrm{~cm}$ ), tendo-se como referência a pressão intragástrica, e tendo como resultado a média das pressões medidas nos quatro canais da sonda, no ponto expiratório médio. Com esse mesmo método se procedeu a determinação da localização e extensão do EIE. 0 relaxamento do EIE foi avaliado pela medida da pressão durante três deglutições de $5 \mathrm{ml}$ de água, com intervalo mínimo de 30 segundos entre elas. A pressão residual foi determinada nesse momento, através da média das três pressões medidas durante 0 período de relaxamento do EIE.

Para registrar as contrações do corpo esofágico, o canal mais distal, dentre os quatro proximais da sonda, foi localizado $3 \mathrm{~cm}$ acima da borda superior do EIE e os outros três a $8 \mathrm{~cm}, 13 \mathrm{~cm}$ e $18 \mathrm{~cm}$ acima do esfíncter, respectivamente. Realizaram-se dez deglutições de $5 \mathrm{ml}$ de água, com intervalo mínimo de trinta segundos entre elas. A amplitude da contração do corpo esofágico foi medida a partir da pressão intraesofágica nos intervalos entre as deglutições e com o paciente respirando normalmente, até o pico da onda de contração. Sua duração foi medida no início da contração até o retorno aos valores de pressão anteriores à contração.

Avaliação da função motora do esôfago. 0 EIE foi avaliado pela observação da extensão, do seu tônus pressórico basal e da sua capacidade de relaxamento durante a deglutição. 0 tamanho do EIE foi considerado normal se entre 3 a 4 centímetros ${ }^{2}$. 0 tônus pressórico basal foi medido pela pressão médio-expiratória e considerado hipotenso se a pressão foi menor que $14 \mathrm{mmHg}$, normotenso quando entre 14 e $35 \mathrm{mmHg}$ e hipertenso quando acima de $35 \mathrm{mmHg}$. A capacidade de relaxamento após cada deglutição foi considerada completa quando sua pressão caiu para níveis inferiores a $4 \mathrm{mmHg}$ ou menores que $20 \%$ da pressão de repouso do EIE $^{20}$.

0 corpo do esôfago foi avaliado pela observação da peristalse e da amplitude da onda de contração. A amplitude de contração foi medida pela média aritmética das pressões de contração obtidas durante as 10 deglutições de água dos canais localizados a 3,8 e $13 \mathrm{~cm}$ acima da borda superior do EIE. Os valores (média $\pm \mathrm{DP}$ ) considerados como referências da normalidade foram de $99 \pm 40 \mathrm{mmHg}$ para o esôfago distal (3 e $8 \mathrm{~cm}$ do EIE) e $109 \pm 45 \mathrm{mmHg}, 90 \pm 41 \mathrm{mmHg}, 70 \pm 32 \mathrm{mmHg}$ para as posições $3,8,13 \mathrm{~cm}$, respectivamente. Considerou-se hipercontratilidade esofágica quando a pressão média do esôfago distal foi maior que 140mmHg e hipocontratilidade esofágica quando menor que $60 \mathrm{mmHg}$. A duração da onda de contração foi determinada pelo tempo médio de duração da contração obtido durante as dez deglutições de água nos canais de leitura. Considerou-se

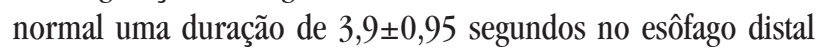

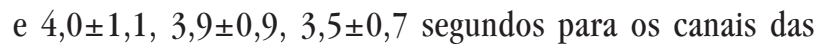
posições $3,8,13 \mathrm{~cm}$, respectivamente ${ }^{20}$. Peristalse primária normal foi definida quando as ondas de contração ocorreram seqüencialmente (assincrônicas) ao longo do traçado, após cada deglutição de água, ordenadas de cima para baixo. Aperistalse completa ocorreu quando observada ausência de ondas de contração esofágica após as dez deglutições de água e aperistalse parcial ou incompleta se observada peristalse normal no esôfago proximal, associada a mais de duas ondas de contração não propagadas e/ou mais de uma onda de contração simultânea em esôfago distal ${ }^{25}$. Foi classificado como distúrbio motor inespecífico (DMI) do corpo do esôfago quando observadas contrações peristálticas e hipocontráteis em relação aos valores médios ou segmentares do corpo do esôfago. Definiram-se como contrações esofágicas simultâneas as ondas de contração que tiveram início simultâneo nos quatro pontos de observação ou se apresentaram velocidade de propagação igual ou maior que $10 \mathrm{~cm} / \mathrm{s}$ acima da pressão intra-esofágica basal. Onda de contração não propagada foi identificada se a pressão intraesofágica se manteve inalterada ou se apresentou uma amplitude abaixo de $15 \mathrm{mmHg}$ após a deglutição e contrações de múltiplos picos quando cada pico de contração teve, no mínimo, 10\% da amplitude média da onda total e com duração mínima de um segundo ${ }^{18}$.

Procedimentos éticos. Este trabalho foi aprovado pelo Comitê de Ética em Pesquisa do Hospital Universitário Júlio Müller, pelo documento número 170/CEP/HUJM/04 de 26/01/2005. A participação no estudo foi voluntária e declarada pelos pacientes em termo de consentimento livre esclarecido.

Análise estatística. Teste de Kruskal-Wallis foi utilizado para determinar diferença entre médias e o teste do $\chi^{2}$ (ou de Fisher) para determinar a significância das associações dicotômicas. Para todos esses testes considerou-se o erro alfa $=0,05$.

\section{RESULTADOS}

Avaliação clínico-laboratorial dos pacientes portadores da forma indeterminada da doença de Chagas crônica. Apesar de não apresentarem manifestações clínicas clássicas, 24 (48\%) dos 50 pacientes incluídos no estudo referiram sintomas digestivos altos por, no mínimo, uma vez por semana nos três meses que antecederam à primeira avaliação. Os sintomas mais freqüentemente relatados por esse grupo foram pirose, desconforto intermitente à deglutição, regurgitação, sialorréia e odinofagia. Quatro (8\%) pacientes referiram desconforto intermitente à deglutição, traduzido como sensação esporádica de opressão retroesternal durante a refeição, com início súbito e resolução espontânea, sem periodicidade e/ou ritmicidade. No entanto, nenhum deles fez menção de dificuldade para deglutição ou qualquer outro sintoma que pudesse ser caracterizado como disfagia. Dos que relataram sialorréia nenhum se declarou etilista. Outras queixas referidas por alguns pacientes foram dor torácica retroesternal atípica, tosse, dispnéia e constipação intestinal crônica (todos com enema opaco normal). Dos pacientes que relataram dispnéia e tosse, nenhum se declarou tabagista e em apenas um a endoscopia digestiva alta revelou refluxo gastroesofágico (Tabela 1).

Resultado do estudo manométrico do esôfago. Nenhum paciente com a FCI apresentou alteração do tamanho do EIE, cujo comprimento, nos 50 pacientes, variou de 2,5 a $4,5 \mathrm{~cm}$, com média (DP) de 3,3 $(0,85) \mathrm{cm}$. Embora a pressão (DP) de repouso do EIE tenha sido de 19,9 $(9,4) \mathrm{mmHg}$, constatou-se 
Tabela 1 - Resultado da avaliação clínica dos 50 pacientes portadores da forma indeterminada da doença de Chagas crônica, atendidos no Ambulatório de Infectologia do Hospital Universitário Júlio Müller, Cuiabá, 2003-2005.

\begin{tabular}{llrr}
\hline Característica & Categoria & Número & Porcentagem \\
\hline Sintomas* $_{(\mathrm{n}=31)}$ & Pirose & 20 & 40 \\
& Desconforto a deglutição & 4 & 8 \\
& Regurgitação & 3 & 6 \\
& Sialorréia & 2 & 4 \\
& Odinofagia & 2 & 4 \\
& Dor retroesternal atípica & 6 & 12 \\
& Tosse & 3 & 6 \\
& Dispnéia & 2 & 4 \\
& Constipação & 5 & 10 \\
& Sem sintomas & 26 & 52 \\
\hline
\end{tabular}

* Alguns pacientes apresentaram mais do que um sintoma

que 14 (28\%) pacientes apresentaram o esfíncter hipotenso e 3 (6\%) apresentaram o esfíncter hipertenso. Os outros 33 (66\%) pacientes apresentaram a pressão do EIE dentro dos limites da normalidade. Observou-se ainda que oito (16\%) pacientes apresentaram relaxamento parcial do EIE à deglutição de água, mantendo uma pressão residual média (DP) de 5,6 $(1,2) \mathrm{mmHg}$ e alterações específicas ou inespecíficas na motilidade do corpo do esôfago (Tabela 2). Nenhum paciente apresentou ausência do relaxamento do EIE após as deglutições de água.
Avaliação da função do corpo do esôfago. Como esperado, nenhum paciente com FCI apresentou aperistalse completa na avaliação do corpo do esôfago. Entretanto, 33 (66\%) pacientes demonstraram alterações no exame manométrico desse segmento, sendo 13 (26\%) classificados como aperistalse parcial e 20 (40\%) com amplitude baixa das contrações esofágicas e, portanto, caracterizados como portadores de DMI.

As pressões e durações médias das contrações do corpo do esôfago medidas nos canais de leitura a 3,8 e $13 \mathrm{~cm}$ acima do EIE estão apresentadas na Tabela 3. A amplitude média (DP) de contração do esôfago distal nos 50 pacientes foi de $90,1(31,8) \mathrm{mmHg}$ e a sua duração média (DP) foi de $4(0,9)$ segundos, valores esses dentro dos limites referenciados para a normalidade. Porém, nos 13 pacientes que apresentaram aperistalse parcial, a amplitude média de contração do esôfago distal foi significativamente menor que a observada nos pacientes peristálticos $(\mathrm{p}<0,05)$, sejam eles portadores de DMI ou com amplitude de contração esofágica normal. Na avaliação da duração da onda de contração não se observou diferença significante entre esses grupos.

Por fim, somente 17(34\%) pacientes apresentaram resultados completamente normais na avaliação manométrica dos dois terços distais do esôfago. Destaca-se que esses pacientes foram significantemente mais jovens $(\mathrm{p}=0,025)$ do que os pacientes portadores de distúrbios da motilidade, tanto específicos quanto inespecíficos (Tabela 2)

\begin{tabular}{|c|c|c|c|c|c|c|c|}
\hline \multirow{2}{*}{$\begin{array}{l}\text { Perfil de motilidade } \\
\text { do esôfago }\end{array}$} & \multirow{2}{*}{$\begin{array}{l}\text { Idade média } \\
\text { (anos) }\end{array}$} & \multicolumn{2}{|c|}{ Sexo } & \multicolumn{2}{|c|}{ EIE } & \multicolumn{2}{|c|}{ Esôfago distal } \\
\hline & & $\begin{array}{l}\text { masculino } \\
\text { (n) }\end{array}$ & $\begin{array}{c}\text { feminino } \\
\text { (n) }\end{array}$ & $\begin{array}{l}\mathrm{PME} \pm \mathrm{DP} \\
(\mathrm{mmHg})\end{array}$ & $\begin{array}{c}\text { relaxamento } \\
\text { incompleto (n) }\end{array}$ & $\begin{array}{l}\text { amplitude média } \\
\pm \mathrm{DP}(\mathrm{mmHg})\end{array}$ & $\begin{array}{l}\text { duração } \pm \mathrm{DP} \\
\text { (segundos) }\end{array}$ \\
\hline Normal & $37,4^{*}$ & 6 & 11 & $21,2 \pm 5,2$ & $0^{* *}$ & $109,8 \pm 16,7^{\text {***** }}$ & $4,1 \pm 0,6$ \\
\hline Alterado & $44,1^{*}$ & 12 & 21 & $19,2 \pm 11,0$ & $8^{2 s}$ & $79,9 \pm 33,1^{\text {****** }}$ & $3,9 \pm 1,0$ \\
\hline DMI & 41,8 & 5 & 15 & $19,6 \pm 12,3$ & 5 & $82 \pm 32,2$ & $4,0 \pm 1,1$ \\
\hline AP & 46,8 & 7 & 6 & $18,7 \pm 9,1$ & 3 & $76,7 \pm 35,4$ & $3,9 \pm 0,8$ \\
\hline Total & 41,8 & 18 & 32 & $19,9 \pm 9,4$ & 8 & $90,1 \pm 31,8$ & $4,0 \pm 0,9$ \\
\hline $\begin{array}{l}\text { EIE: esfíncter inferiol } \\
\text { DP: Desvio padrão } \\
* \mathrm{p}=0,019\end{array}$ & $\begin{array}{r}\text { sôfago PME: } \\
\text { AP: ap } \\
* * \mathrm{p}=\end{array}$ & $\begin{array}{l}\text { ressão médi } \\
\text { ristalse parc } \\
, 025 \text { (Fishe }\end{array}$ & $\begin{array}{l}\text {-expiratória } \\
\text { l }\end{array}$ & RI: relaxam & nto incompleto & II: distúrbio motor & specífico \\
\hline
\end{tabular}

Tabela 3 - Amplitude e duração médias das contrações esofágicas, medidas em diferentes posições acima da borda superior do esfíncter inferior do esôfago, após deglutições de água, de pacientes portadores da forma crônica indeterminada da doença de Chagas.

\begin{tabular}{|c|c|c|c|c|c|c|c|c|}
\hline \multirow{3}{*}{$\begin{array}{l}\text { Perfil de motilidade do } \\
\text { esôfago (n) }\end{array}$} & \multicolumn{8}{|c|}{ Posição do canal de leitura da pressão do corpo do esôfago } \\
\hline & \multicolumn{2}{|c|}{$3 \mathrm{~cm}$} & \multicolumn{2}{|c|}{$8 \mathrm{~cm}$} & \multicolumn{2}{|c|}{$13 \mathrm{~cm}$} & \multicolumn{2}{|c|}{$3+8 \mathrm{~cm}$} \\
\hline & $\begin{array}{l}\text { amplitude } \\
(\mathrm{mmHg})\end{array}$ & $\begin{array}{c}\text { duração } \\
(\text { seg })\end{array}$ & $\begin{array}{l}\text { amplitude } \\
(\mathrm{mmHg})\end{array}$ & $\begin{array}{c}\text { duraçãa } \\
(\text { seg })\end{array}$ & $\begin{array}{l}\text { amplitude } \\
(\mathrm{mmHg})\end{array}$ & $\begin{array}{c}\text { duração } \\
(\text { seg })\end{array}$ & $\begin{array}{l}\text { amplitude } \\
(\mathrm{mmHg})\end{array}$ & $\begin{array}{r}\text { duração } \\
(\text { seg })\end{array}$ \\
\hline Aperistalse parcial (13) & $88,1(38,9)$ & $4,2(1,3)$ & $65,2(34,1)$ & $3,6(0,9)$ & $28,5(13,5)$ & $2,5(0,3)$ & $76,7(35,4)$ & $3,9(0,8)$ \\
\hline Peristalse (37) & $106,2(37,6)$ & $4,1(1,1)$ & $86,3(22,9)$ & $3,9(0,9)$ & $50,9(20)$ & $3,0(0,6)$ & $81,7(29,7)$ & $4,0(0,8)$ \\
\hline normal (17) & $123,6(28,3)$ & $4,3(0,8)$ & $96,0(13,1)$ & $3,9(0,5)$ & $51,0(16,8)$ & $3,0(0,7)$ & $109,8(16,7)$ & $4,1(0,6)$ \\
\hline DMI (20) & $88,4(39,0)$ & $4,0(1,3)$ & $75,6(27,2)$ & $3,9(1,2)$ & $49,6(22,8)$ & $3,0(0,8)$ & $82,0(32,2)$ & $3,9(1,1)$ \\
\hline \multicolumn{9}{|l|}{ Valor de $\mathrm{p}$} \\
\hline (aperistalse parcial $v s$ peristalse) & 0,14 & 0,68 & 0,007 & 0,32 & 0,0004 & 0,049 & 0,042 & 0,68 \\
\hline Total (50) & $101,4(38,4)$ & $4,2(1,1)$ & $80,9(27,6)$ & $3,8(0,9)$ & $44,6(20,8)$ & $2,9(0,8)$ & $91(31,3)$ & $4(0,9)$ \\
\hline
\end{tabular}

DMI: distúrbio motor inespecífico 
Associação entre alterações motoras esofágicas e sintomas do trato digestivo superior. Entre os 33 (66\%) pacientes que apresentaram alterações no exame manométrico do esôfago, 17 (51,5\%) referiram sintomas do trato digestivo superior. Este grupo incluiu os quatro pacientes que referiram desconforto intermitente à deglutição, cujas alterações manométricas foram aperistalse parcial em um e DMI em três pacientes. Por outro lado, dos 17 indivíduos com exame manométrico normal, sete $(41,2 \%)$ referiram sintomas digestivos altos. Essa diferença, contudo, não foi estatisticamente significante $(p=0,69)$. Destaca-se, nesta análise, a alta $(48,5 \%)$ frequiência de indivíduos assintomáticos apresentando distúrbios motores do esôfago (Tabela 4). A média (DP) da idade desse grupo de pacientes foi de 39,8 $(11,2)$ anos.

Tabela 4 - Relação entre sintomas do trato digestivo superior inespecíficos ou alteração endoscópica e distúrbio motor do esôfago em pacientes portadores da forma crônica indeterminada da doença de Chagas, atendidos no Ambulatório de Infectologia do Hospital Universitário Júlio Mïller, 2003 a 2005.

\begin{tabular}{|c|c|c|c|c|c|c|c|}
\hline \multirow[t]{3}{*}{$\begin{array}{l}\text { Sintomas do trato } \\
\text { digestivo superior }\end{array}$} & \multirow{3}{*}{$\begin{array}{c}\text { Idade } \\
\text { (média } \pm \mathrm{DP}) \\
(\text { anos })\end{array}$} & \multicolumn{4}{|c|}{$\begin{array}{c}\text { Distúrbio motor do } \\
\text { esôfago }\end{array}$} & \multirow[t]{3}{*}{ Total } & \multirow[t]{3}{*}{$\begin{array}{l}\text { Valor } \\
\text { de } p\end{array}$} \\
\hline & & \multicolumn{2}{|c|}{ presente } & \multicolumn{2}{|c|}{$\overline{\text { ausente }}$} & & \\
\hline & & $\mathrm{n}^{0}$ & $\%$ & & $\%$ & & \\
\hline Sim & $41 \pm 10,2$ & 17 & 51,5 & 7 & 41,2 & 24 & \\
\hline Não & $42,6 \pm 11,2$ & 16 & 48,5 & 10 & 58,8 & 26 & 0,69 \\
\hline Total & $41,8 \pm 10,6$ & 33 & 66,0 & 17 & 34,0 & 50 & \\
\hline \multicolumn{8}{|c|}{ Alteração endoscópica } \\
\hline Sim & $45,2 \pm 8,5$ & 7 & 41,2 & 3 & 42,9 & 10 & \\
\hline Não & $39,6 \pm 11,9$ & 10 & 58,8 & 4 & 57,1 & 14 & 0,64 \\
\hline Total & $41,9 \pm 10,9$ & 17 & 70,8 & 7 & 29,2 & 24 & \\
\hline
\end{tabular}

DP: desvio padrão

0 resultado da endoscopia digestiva alta dos 24 pacientes sintomáticos mostrou que 10 (41,7\%) eram portadores de algum tipo de lesão da mucosa gastro-esofágica, diagnosticadas como esofagite, gastrite, hérnia hiatal e úlcera gástrica. Sete (70\%) deles eram portadores de distúrbio motor do esôfago. Essa frequiência de alteração motora não foi diferente da observada nos indivíduos com endoscopia digestiva alta normal $(\mathrm{p}=0,64)$. Mesmo excluindo os pacientes portadores de esofagite e hérnia hiatal ao exame endoscópico, a frequiência de alterações motoras encontradas, sejam elas inespecíficas ou aperistalse parcial, permaneceu elevada (68\%). Nenhuma alteração endoscópica foi constatada nos pacientes que relataram desconforto intermitente a deglutição.

\section{DISCUSSÃO}

Nestes tempos de controle da transmissão da doença de Chagas e da ocorrência de formas excepcionais de transmissão em áreas anteriormente não endêmicas, o estudo da FCI é uma prioridade para os interessados nos problemas causados pela doença ${ }^{1}$. No presente estudo, quase a metade dos pacientes com FCI referiu algum tipo de sintoma digestivo alto, que não a disfagia. Uma vez que co-morbidades indutoras de lesão esofágica foram descartadas, a presença desses sintomas pode sugerir acometimento esofágico da doença, como preconizam alguns autores ${ }^{27}$. Assim, o relato de queixas inespecíficas nesses pacientes tem significado controverso e pode exigir a realização de exames específicos, para revelar ou não o acometimento de órgão alvo e, por conseguinte, auxiliar a classificação clínica do paciente ${ }^{22}$.

É sabido que pacientes com doença de Chagas apresentam distintos graus de comprometimento motor ao longo de todo o tubo digestivo ${ }^{21}$ e diversos trabalhos já demonstraram alterações motoras do esôfago, mesmo em indivíduos assintomáticos ${ }^{911}$. Alguns desses achados manométricos já foram descritos como sendo consequiência do comprometimento neuronal da esofagopatia chagásica ${ }^{25}$. No presente estudo, foi identificado importante (66\%) parcela dos pacientes portadores da FCI com algum grau de comprometimento motor do esôfago na manometria. Embora tenham predominado alterações inespecíficas, $26 \%$ dos pacientes exibiram comprometimento da coordenação da contração do esôfago distal ou do relaxamento do EIE, descritos como sugestivos de esofagopatia chagásica ${ }^{25}$. Esse achado corrobora a controvérsia sobre a conceituação sistematizada da forma indeterminada da doença de Chagas crônica, uma fez que é muito difícil definir normalidade apenas com os recursos que são propostos para essa definição ${ }^{1625}$.

Apesar de alguns estudos já tenham demonstrado alteração da coordenação peristáltica e diminuição da amplitude média de contração do esôfago distal em pacientes chagásicos sintomáticos e assintomáticos ${ }^{9125}$, pouca informação existe sobre as medidas manométricas do corpo do esôfago em pacientes portadores da FCI ${ }^{3}$. Como esperado, a avaliação manométrica do corpo do esôfago não revelou aperistalse completa do órgão entre os pacientes aqui estudados. Todavia, aperistalse parcial foi achado freqüente na avaliação desse segmento. Além disso, observouse menor amplitude de contração esofágica nos pacientes portadores de aperistalse parcial, em relação aos grupos com peristalse normal ou com DMI. Estes achados sugerem que 0 comprometimento neuronal do esôfago distal está presente na FCI, como já demonstrado anteriormente para indivíduos chagásicos sintomáticos ${ }^{25}$.

A pressão do EIE está diminuída, normal ou aumentada em pacientes chagásicos assintomáticos e sintomáticos ${ }^{92} 24$. Vários estudos já demonstraram alterações não significativas na pressão média de repouso do EIE em pacientes com peristalse e aperistalse na doença de Chagas e não observaram relação entre alterações na função do EIE e presença de sintomas como disfagia e regurgitação $0^{4}$. Da mesma forma, o presente estudo não mostrou diferença estatística entre a pressão média expiratória do EIE nos grupos com peristalse normal, aperistalse parcial ou DMI.

Alterações manométricas no esôfago distal também podem ser observadas em pacientes com esofagite, principalmente em casos conseqüentes a doença do refluxo gastro-esofágica grave, bem como em outras doenças que causam prejuízo da inervação e/ou da capacidade de contração muscular do esôfago, inclusive doença de Chagas?. Uma vez que foram afastadas as 
principais co-morbidades que podem confundir a interpretação da contratilidade do esôfago distal, tais como as colagenoses, diabetes mellitus, alcoolismo e a hérnia de hiato diafragmático, é provável que as alterações observadas neste estudo estejam sendo provocadas pela lesão neuronal chagásica. Além disso, a ausência de lesão mucosa ao estudo endoscópico do esôfago nos pacientes portadores de DMI ou com aperistalse incompleta sugere que a diminuição da amplitude da contração esofágica, bem como as alterações funcionais do EIE, possam ser conseqüentes à destruição da inervação colinérgica, presentes nos pacientes portadores da doença de Chagas?

A alta (48,5\%) frequiência de distúrbios motores do esôfago em indivíduos assintomáticos deste estudo, principalmente com aperistalse parcial, leva ao questionamento da possibilidade de sua ocorrência em indivíduos saudáveis e assintomáticos. Embora alterações manométricas em voluntários saudáveis já tenham sido observadas em vários estudos que utilizaram grupos controles assintomáticos, em todos eles os achados compatíveis com o que se classificou como DMI no presente trabalho foram relatados em menos de $20 \%$ e como aperistalse parcial em menos de $9 \%$ dos voluntários ${ }^{79} 15192029$

Outro aspecto a ser considerado é o efeito da idade sobre a integridade motora do esôfago, principalmente após os 60 anos, como já demonstrado em outros estudos ${ }^{5}$. Meneghelli e cols. ${ }^{23}$ observaram que a lesão esofágica progride com os anos em quase $50 \%$ dos pacientes chagásicos, independente da forma clínica da doença ou da existência de lesão esofágica e disfagia prévias. Embora essa progressão possa ser resultante de lesão neural chagásica, questiona-se se o próprio processo de envelhecimento da pessoa pode estar interferindo na capacidade motora do esôfago desses pacientes, semelhante ao observado neste estudo, onde as alterações motoras também foram menos frequientes entre os pacientes mais jovens ${ }^{23}$. No entanto, os indivíduos assintomáticos que apresentaram as alterações acima discutidas eram todos adultos jovens e com idade inferior à relatada nesses estudos como associadas à modificação senil do esôfag $0^{5}{ }^{10}$. Portanto, a maior frequiência observada de DMI e aperistalse parcial nesse grupo pode ser consequiência de desnervação chagásica e coloca em perspectiva as implicações potenciais dessas anormalidades como sugestivas de sinais preditivos de comprometimento esofágico em pacientes com FCI.

Não obstante a grande frequiência de queixas digestivas altas referidas pelos portadores da FCI, não se observou associação entre sintomas e presença de distúrbio motor do esôfago à manometria, seja na avaliação do EIE como na do corpo do esôfago. De fato, essa associação só foi identificada em estudos que incluíram pacientes disfágicos e com alteração no corpo do esôfag $0^{469}{ }^{14}$, sugerindo que a disfagia é o único sintoma que pode ser considerado preditor de alteração precoce da inervação do esôfag $0^{1425}$. É ainda possível que 0 número de pacientes aqui avaliados não tenha sido suficiente para mostrar alguma diferença.

Uma explicação para os sintomas do trato digestivo superior referidos pelos pacientes seria a presença de lesões inflamatórias na mucosa gástrica e/ou esofágica. Ou ainda que a presença delas estivesse provocando a disfunção motora e com isto confundindo a análise da associação entre sintomas e alterações manométricas do esôfago. Na verdade, quase metade dos indivíduos sintomáticos teve alterações no exame endoscópico alto do tubo digestivo. Porém essas lesões endoscópicas não diferiram entre os grupos com e sem alterações motoras do esôfago desses pacientes.

Em conclusão, os portadores da FCI aqui estudados apresentam sintomas digestivos altos inespecíficos que podem dificultar a sua classificação evolutiva com base apenas no exame clínico e radiológico. Embora não se tenha demonstrado associação entre sintomas do trato digestivo superior e distúrbio motor do esôfago em pacientes com FCI, é alta a frequiência de alterações motoras do esôfago entre esses pacientes, sejam elas específicas (aperistalse parcial e relaxamento incompleto do EIE) ou inespecíficas (hipocontratilidade).

\section{REFERÊNCIAS}

1. Andrade ZA. A forma indeterminada da doença de Chagas em tempos de controle do Triatoma infestans. Revista de Patologia Tropical 34:105-111, 2005.

2. Corsi PR, Gagliardi D. Detalhes técnicos da execução do exame. In Nasi A, Michelsohn NH (eds) Avaliação Funcional do Esôfago. Editora Roca, São Paulo, p. 25-36, 2001

3. Crema E, Ribeiro L, Cruvinel L, Werneck A, Oliveira R, Silva A. Estudo eletromanométrico do esôfago em portadores da doença de Chagas em sua forma indeterminada. Revista da Sociedade Brasileira de Medicina Tropical 38 (supl):493, 2005.

4. Dantas R0. Relation between motility and esophageal symptoms and lower esophageal sphincter pressure in Chagas'disease. Gastroenterologia Endoscopia Digestiva 12:23-26, 1993.

5. Dantas R0. Motilidade do esôfago no paciente com mais de 70 anos. Arquivos de Gastroenterologia 32:3-6, 1995.

6. Dantas RO. Dysphagia in patients with Chagas'disease. Dysphagia 13:53-57, 1998.

7. Dantas RO. Hipocontratilidade do esôfago em pacientes com doença de Chagas e pacientes com acalasia idiopática. Arquivos de Gastroenterologia 37:35-41, 2000.

8. Dantas RO. Effect of successive swallows on oesophageal motility of normal volunteers, patients with Chagas'disease and patients with idiopathic achalasia. Neurological Gastroenterology Motility 15:57-62, 2003.

9. Dantas Ro. Motilidade do esôfago no paciente com doença de Chagas sem megaesôfago. Gastroenterologia Endoscopia Digestiva 22:79-84, 2003.

10. Dantas RO, Ciscato Júnior JG. Influence of age in esophagus involvement in Chagas' disease. Gastroenterologia e Endoscopia Digestiva 16:1-5, 1997.

11. Dantas RO, Deghaide NHS, Donadi EA. Esophageal manometric and radiologic findings in asymptomatic subjects with Chagas'disease. Journal of Clinical Gastroenterology 28:245-248, 1999.

12. Dantas R0, Godoy RA. Pressão de repouso do esfíncter inferior do esôfago na esofagopatia chagásica. Medicina 12:1-5, 1980.

13. Dantas RO, Godoy RA, Padovan W, Meneghelli UG, Oliveira RB. The esophageal contractility in Chagas'disease. Gastroenterologia Endoscopia Digestiva 1:26-28, 1983.

14. Dantas RO, Modena JLP, Bellucci AD. Relation between dysphagia and esophageal endoscopic, manometric and radiologic findings in patients with chagas'disease. Gastroenterologia Endoscopia Digestiva 8:13-16, 1989.

15. Flores PP, Lemme EMO, Coelho HSM. Alterações da motilidade esofagiana em pacientes cirróticos com varizes de esôfago não submetidos a tratamento endoscópico. Arquivos de Gastroenterologia 42:213-220, 2005.

16. Ianni BM, Mady C. A Forma Indeterminada da Doença de Chagas. Mitos vs Fatos. Arquivos Brasileiros de Cardiologia 68:147-148, 1977. 
17. Köberle F. Chagas'disease and Chagas' syndrome: The pathology of American Trypanossomiasis. Advences in Parasitology 6:63-116, 1968.

18. Lemme EMO. Métodos de avaliação e interpretação. In: Nasi A, Michelson $\mathrm{NH}$ (eds) Avaliação Funcional do Esôfago, Editora Roca, São Paulo, p. 37-44, 2001.

19. Lemme EMO, Domingues GR, Silva LF, Firman CG, Pantoja JAS. Esofagomanometria computadorizada: resultados preliminares em voluntários adultos saudáveis. GED Gastroenterologia Endoscopia Digestiva 20:29-35, 2001

20. Lobato LC, Castell DO. Filho JR. Função motora normal do esôfago. In: Nasi A, Michelson NH (eds) Avaliação Funcional do Esôfago. Editora Roca, São Paulo, p. $45-60,2001$

21. Madri AM, Quera R, Delfilippi C, Gil LC, Sapunar P, Henriquez A. Gastrointestinal motility disturbances in Chagas disease. Revista Médica de Chile 132: 939-946, 2004

22. Manzullo EC, Darraidou MA, Libonatti O, Rozlosnik J, Bazzano AC. Estudo longitudinal de la cardiopatia chagásica crônica. Centro de Chagas de la Cátedra de Enfermedades Infecciosas de la Faculdad de Ciências Médicas de Buenos Aires, p1-141, 1982.

23. Meneghelli UG, Peria FM, Darezzo FM, Almeida FH, Rodrigues CM, Aprile LR, Dantas R0. Clinical, radiographic, and manometric evolution of esophageal involvement by Chagas' disease. Dysphagia 20:40-45, 2005.
24. Moraes Filho JPP, Kahatsu OS, Batarello A. Pressão basal do esfíncter inferior do esôfago na doença de Chagas: Megaesôfago e forma indeterminada. Revista da Associação Médica Brasileira 32:51-53, 1986.

25. Oliveira RB, Rezende-Filho J, Dantas RO, Iazigi N. The spectrum of esophageal motor disorders in Chagas'disease. American Journal of Gastroenterology 90:1119-1124, 1995

26. Reunião de Pesquisa Aplicada em Doença de Chagas. Validade do conceito de forma crônica indeterminada de doença de Chagas. Revista da Sociedade Brasileira de Medicina Tropical 18:46, 1985.

27. Rezende JM, Moreira H. Forma Digestiva da Doença de Chagas. In: Brener Z, Andrade ZA, Barral-Neto M (eds) Trypanosoma cruzi e doença de Chagas. $2^{\text {nd }}$ edição. Guanabara-Koogan, Rio de Janeiro, p. 297-343, 2000

28. Rezende-Filho J. Estudo cintilográfico do trânsito esofagiano na esofagopatia chagásica crônica. Dissertação de Mestrado, Faculdade de Medicina de Ribeirão Preto, São Paulo, SP, 1985.

29. Ricther JE, Wu WC, Johns DN, Blackwell JN, Nelson JL 3rd, Castell JA, Castell DO. Esophageal manometry in 95 healthy adult volunteers. Variability of pressures with age and frequency of "abnormal" contractions. Digestive Diseases and Sciences 32:583-592, 1987.

30. Tarleton RL. Chagas'disease: a role for autoimmunity. Trends in Parasitology 19:447-451, 2003 